Bioscientia Medicina: Journal of Biomedicine \&

Translational Research

Journal Homepage: www.bioscmed.com

\title{
NFATc1 and Tumor Associated Macrophages Affect Progression of Certain Subsets of Diffuse Large B Cell Lymphoma Non-GCB Subtypes
}

\author{
Krisna Murti ${ }^{\star}$, Muslina ${ }^{1}$, Ika Kartika $^{1}$, Rachmat Hidayat $^{2}$ and Ella Amalia ${ }^{3}$
}

${ }^{1}$ Department of Anatomic Pathology, Faculty of Medicine, Universitas Sriwijaya Palembang, Indonesia 2Department of Biology, Faculty of Medicine, Universitas Sriwijaya Palembang

${ }^{3}$ Department of Microbiology, Faculty of Medicine, Universitas Sriwijaya Palembang

\section{A R T I C L E I N F O Keywords: \\ Diffuse large B cell lymphoma NFATc1 \\ MYC \\ PD-L1 \\ CD163 \\ *Corresponding author: \\ Krisna Murti \\ E-mail address: \\ krisna.arinafril@unsri.ac.id}

All authors have reviewed and approved the final version of the manuscript.

\begin{abstract}
A B S T R A C T
Background: Diffuse large B cell lymphoma (DLBCL) is the most common type of non-Hodgkin lymphoma among B cell lymphomas. The interaction of tumor cells with their microenvironment (tumor microenvironment, TME) leads to progressivity of malignancy. $\mathrm{CD} 163^{+}$macrophages known as components of TME. Nuclear factor of activated T cell (NFATc1) and MYC are important transcription factors in malignant transformation and progression. Therapeutic strategies were fast developed, nevertheless, efforts to decrease DLBCL morbidity and mortality are unsatisfied, therefore,new markers for prognosis and or therapeutic options of the patients are necessary. This study was aimed to investigate NFATc 1 expression in DLBCL and its TME. Methods: Thirty-two paraffin blocks were selected then immunostained for expression of NFATC1, MYC, and CD163. Clinopathologic data i.e. ages, gender, and proliferation index Ki-67 were obtained. Data was analyzed by statistics Result: Positive expression of CD163 and NFATc1 was among 55\% and $45 \%$ of cases respectively. All DLBCL cases in this study were non-GCB subtype and more patients were under 60 years $(66 \%)$. Positive expression of CD163 was higher in males (69\%) and in patients under 60 years $(63 \%)$. Tissues positive for both NFATc1 and CD163 was observed higher among males and patients under 60 years. Conclusion: NFATc 1 may affect development and or progression of certain subsets of DLBCL non-GCB subtype.
\end{abstract}

https://doi.org/10.32539/bsm.v5i3.136

\section{Introduction}

Diffuse large B-cell lymphoma (DLBCL) is a neoplasm with a diffuse growth pattern and the individual cells have medium or large B lymphoid cells with nuclei are the same size as, or larger than, those of normal macrophages, or more than twice the size of those of normal lymphocytes. ${ }^{1}$ The incidence of DLBCL tends to increase every year based on unpublished data from our department. According to Hans' algorithm DLBCL classified into germinal centre $B$ cells (GCB) and non-germinal centre B cells (non-GCB) subtypes. Patients with the GCB subtype usually have better prognosis than patients with the non-GCB subtype.

Interaction of tumor cells with their microenvironment (tumor microenvironment, TME) leads to progressivity of malignancy. Some components contribute to TME i.e. immune check point, which is activated through the interaction of programmed death-1 (PD-L) and its ligands PD-L1 and or PD-L2.3 Tumor associated macrophages (TAMs) with phenotype M2-like macrophages, can express crucial molecules of immune checkpoint, including programmed cell death protein 1 (PD-1) and programmed cell death-ligand 1/2 (PD-L1/2), hence, inhibiting the overall inflammatory response, permitting the evasion of the tumor cells to antitumor immunity. 4,5

Nuclear factor of activated T cell (NFATc1) is an 
important transcription factor in physiologic mechanisms of several cell types and organs as well as in pathogenesis of certain diseases including malignant transformation and tumor progression. ${ }^{6}$ It is also known has roles in regulation of $\mathrm{PD}-1$ activation. 7 The examination of NFATc1 expression also contributes to our understanding of the oncogenic pathways involved in DLBCL. Therapeutic strategies for lymphomas including DLBCL were fast developed, from standard regiment by $\mathrm{R}-\mathrm{CHOP}$ and introduction of immunotherapy, ${ }^{8}$ yet, still treatment failure was observed, therefore, new markers for prognosis or therapy of the patients are necessary.

Dr. Mohammad Hoesin Hospital Palembang, is a referral hospital for patients all over South Sumatera. Among non-Hodgkin lymphomas DLBCL cases shows the highest incidence in these populations, however, studies and data correlated to tumor microenvironment of DLBCL are significantly limited. The purpose of this study is to investigate the roles of NFATc1 expression in DLBCL and its TME. We will explore whether NFATc1 staining can be used as a useful immunohistochemical complement that could potentially determine DLBCL prognosis or therapy by exploring the NFATc1 staining profile in this lymphoma.

\section{Materials and methods}

Patient data

We reviewed surgical pathology reports of patients with DLBCL diagnostic period was from 2016 to 2017. Thirty-two paraffin blocks were selected; consisting of DLBCL (29) and 3 blocks were as controls, which consisting of two cases of B-cell lymphoma, unclassifiable (BCLU) and one case of Burkitt lymphoma. Clinopathologic data i.e. ages, gender, and immunohistochemistry profile including proliferation index Ki-67 were obtained.

\section{Immunohistochemistry}

The representative formalin-fixed paraffinembedded (FFPE) tissue blocks of DLBCL, BCLU and $\mathrm{BL}$ cases were selected and retrieved from the archives of the Department of Anatomic Pathology, Faculty of Medicine Universitas Sriwijaya. Immunohistochemical staining was performed using manual system according to standard immunohistochemical protocol. The analyses were validated using appropriate negative and positive controls by using several tissue blocks consisting of tonsil, appendix, melanoma and tissues.

The blocks were sectioned with a standard microtome (Thermo, Shandon, finesse 325) at $4 \mu \mathrm{m}$ thickness. Subsequently, the slides were dried in a lab heating and drying oven (Sharp, Japan) at power level 8 for $10 \mathrm{~min}$. The tissue sections were deparaffinized in xylene and rehydrated by sequential incubation in ETOH/water solutions. Antigen retrieval was performed by treating the slides in a microwave in citrate buffer. The tissue sections were rinsed in PBS after antigen retrieval procedure and incubated with peroxidase blocking solution for $20 \mathrm{~min}$ at RT. After three washing steps in PBS (5 min each) the tissues were incubated with primary antibody anti-NFATc1 (clone 7A6, dilution 1:100, Abcam, Cambrigde, MA), anti-MYC (clone Y69 rabbit monoclonal, dilution 1:100, Abcam, Cambrigde, MA), and anti-CD163 (clone 10D6, rabbit, monoclonal, dilution 1:100, thermo fisher, USA). Each primary antibody was incubated for 60 minutes at RT, washed thrice and incubated with ADVANCE HRP LINK for 20 min at RT. After three more washing steps the slides were incubated with ADVANCE HRP enzyme for $20 \mathrm{~min}$ at $\mathrm{RT}$ and rinsed three times in PBS. For visualization the tissue sections were incubated with $\mathrm{DAB}$ for $20 \mathrm{~min}$ at RT followed by background staining with Mayer's Hematoxylin for 3-5 min at RT. The slides then were rinsed in running tap water for $5 \mathrm{~min}$, dehydrated through 95\% ethanol for $2 \mathrm{~min}$, cleared in xylene for 2x5 min at RT and finally covered with mounting medium and coverslips. Tissues are analyzed and pictures'acquiring using Olympus DP2 1 U-TV0.5XC-3 SN OHO7164 T2 (Tokyo, Japan) camera at a $\times 400$ magnification.

Expression of NFATc1, CD163, and MYC 
Despite of their intensity either weak, intermediate or strong staining, the positivity of the all antibodies were counted, because the inconsistency of tissue fixation and processing was most likely influencing the intensity of staining. NFAT and MYC positive expression was determined in nuclei of tumor cells as well as in lymphocytes and macrophages surrounding tumors. The expression of PD-L1 was counted in membrane of tumor cells and macrophages around tumor cells, whereas the expression of CD163 was calculated in membrane and or cytoplasm of macrophages around tumor cells. Image $\mathrm{J}$ was used to calculate the numbers of protein expression of NFATc1, CD163, and MYC. In addition, Ki-67 expression was grouped into very high if its counting was more than $70 \%$ and was considered high if that was between 40-70\%.9,10

MYC was considered positive overexpression if the expression of MYC was found in more than $40 \%$ among tumor cells, and vice versa as previously reported (Johnson et al., 2012). While for CD163 and NFATc 1 the classification to be positive or negative was as follow; at first under low power field (100x) the densest five areas were chosen, then among these areas, five locations under high magnification (400x) of the densest focuses were selected. By using image $J$ the all cells expressed either NAFTc1, or CD163 were calculated and noted. Of these 5 areas, the average was counting by using excel. The median of all samples of each antibody was considered as a cut-off point for calculation of NAFTc1, or CD163, or PD-L1 expression. The expression of CD163 or NFATc1 was considered positive if the average of each sample correlated to that antibodies was above those cut-off point and vice versa.

\section{Statistical analysis}

The correlation of expression of NFATc1 with CD163 or MYC or Ki67 was analyzed by the chi square test from SPSS version 20.0 software. P values under 0.05 were considered to be statistically significant.

\section{Results}

\section{Patient data}

Of 32 cases, DLBCL were 29 and 3 cases were controls, which consisting of two cases of B-cell lymphoma, unclassifiable (BCLU) and one case of Burkitt lymphoma (BL). Patients with more than $70 \%$ Ki 67expression were observed among 59\% DLBCL. BCLU and BL showed high expression of $\mathrm{Ki}-67$ in which almost $100 \%$ for both types of lymphomas and all the three cases were also found have overexpression of MYC (table 1). As can be seen from table 1, DLBCL patients in this study were more under 60 years $(66 \%)$ with the mean age was 54 years (Mean $\pm \mathrm{SD}=54.03 \pm 12.95)$ and the median age was 55 years (range: 28-88 years). Our study showed male patients (59\%) were slightly more than females (41\%), with the gender ratio (M: F) was 1.2:1. Nodal location (56\%) was slightly higher than extra nodal. The extra nodal locations were tonsils (3 cases), oropharynx ( 1 cases), nasopharynx (1 cases), femur (1 cases), frontal (1 cases), mesoileum (1 cases), ovarium (1 cases), ileumcolon ( 1 cases), and an intraabdominal case.

\section{Morphological variants}

The cellular morphology of our samples was heterogeneous (Figure 1), but it was dominated by centroblastic variant (18 out $29 ; 62.1 \%)$. Immunoblastic variant was detected in 5 out 29 cases $(17.2 \%)$, while anaplastic variant was observed in 2 cases $(6.9 \%)$ and the rare variant was found in 4 cases $(13.8 \%)$.

Immunohistochemistry of NFATc1, CD163, and MYC.

Positive expression of NFATc1 in the nuclei of tumor cells was observed higher in younger patient (under 60 years, 9 out 13; 69\%). However, we did not see nuclear expression of NFATc1 in macrophages $\left(\mathrm{CD}^{163^{+}}\right)$as well as in small lymphocytes in TME. In these small lymphocytes the expression of NFATc1 were observed more in their cytoplasm. Meanwhile, Positive expression of CD163 was detected higher in males (11 out of $16 ; 69 \%$ ) and in younger patients (under 60 years, 10 out 16; 63\%). Positivity of both NFATc1 and CD163 was observed higher among males 
and younger patients. MYC overexpression was confirmedin55\% DLBCL cases, and was found more among younger subjects (under 60 years, 11 out 16;
$69 \%)$. Statistically, neither significant correlation of NFATc1 to CD163 nor MYC (table 2)

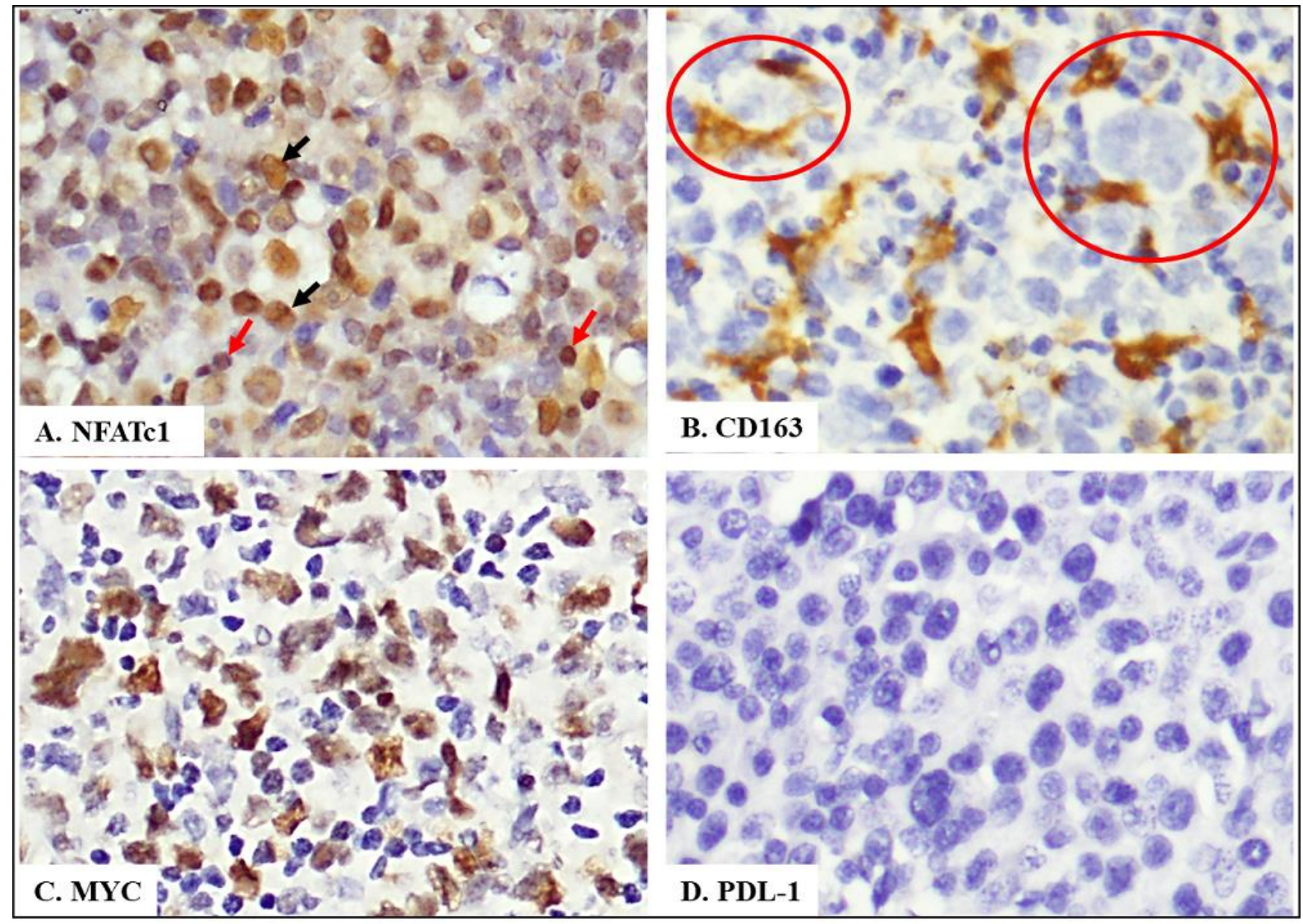

Figure 1. Representative immunolabelling of NFATc1 (A), CD163 (B), MYC (C) and PD-L1 (D) in DLBCL samples. Immunoreactivity of NFATc1 (A, black arrow) and MYC (C) were detected in nuclei of tumor cells. NFATc1 was not observed in macrophages, but only shown in nuclei (red arrow) of some lymphocytes of TME (A). Immunoreactivity of CD163 was detected in membrane and or cytoplasmic of M2 macrophages; note the 2-3 macrophages encircle anaplastic DLBCL tumor cells (B, red circle). (A-D) Original magnification of 400x.

Table1. Clinicopathologic characteristic and expression of NFATc1, Ki-67, MYC, CD163.

\begin{tabular}{|c|c|c|c|c|c|c|c|c|c|}
\hline \multirow{2}{*}{\multicolumn{2}{|c|}{$\begin{array}{c}\text { Clinicopathologic Ch } \\
\begin{array}{c}\text { aracteristics } \\
\text { n=32 (\%) }\end{array}\end{array}$}} & \multicolumn{2}{|c|}{$\begin{array}{l}\text { Ki-67, } \\
\text { n (\%) }\end{array}$} & \multicolumn{2}{|c|}{$\begin{array}{l}\text { MYC, } \\
\text { n (\%) }\end{array}$} & \multicolumn{2}{|c|}{$\begin{array}{c}\text { NFATc1, } \\
\text { n (\%) }\end{array}$} & \multicolumn{2}{|c|}{$\begin{array}{c}\text { CD163, } \\
\text { n (\%) }\end{array}$} \\
\hline & & Very high & High & Pos & Neg & Pos & $\mathrm{Neg}$ & Pos & Neg \\
\hline \multicolumn{10}{|l|}{ Gender } \\
\hline \multirow[t]{2}{*}{ Male } & $17 *$ & $9(53)$ & 8 & 6 & 11 & 8 & 9 & 11 & 6 \\
\hline & (59) & & $(47)$ & (35) & (55) & $(47)$ & (53) & (65) & (35) \\
\hline \multirow[t]{2}{*}{ Female } & 12 & 5 & 7 & 10 & 2 & 5 & 7 & 5 & 7 \\
\hline & (41) & $(42)$ & (58) & (83) & (17) & $(42)$ & (58) & $(42)$ & (58) \\
\hline \multicolumn{10}{|l|}{ Age (years) } \\
\hline$<60$ & $19 *$ & 9 & 10 & $11^{*}$ & 8 & 9 & 10 & 10 & 9 \\
\hline
\end{tabular}




\begin{tabular}{cccccccccc} 
& $\mathbf{( 6 6 )}$ & $(47)$ & $(53)$ & $(58)$ & $(42)$ & $(47)$ & $(53)$ & $(53)$ & $(47)$ \\
$\geq \mathbf{6 0}$ & $\mathbf{1 0}$ & 6 & 4 & 5 & 5 & 4 & 6 & 6 & 4 \\
& $\mathbf{( 3 4 )}$ & $(60)$ & $(40)$ & $(50)$ & $(50)$ & $(40)$ & $(60)$ & $(60)$ & $(40)$ \\
\hline Location & & & & & & & & & \\
\hline Nodal & $\mathbf{1 8}$ & 7 & 11 & 8 & 10 & 6 & 12 & 12 & 6 \\
& $\mathbf{( 6 2 )}$ & $(39)$ & $(71)$ & $(44)$ & $(56)$ & $(33)$ & $(77)$ & $(67)$ & $(33)$ \\
Extra nodal & $\mathbf{1 1}$ & 7 & 4 & 8 & 3 & 7 & 4 & 4 & 7 \\
& $\mathbf{( 3 8 )}$ & $(64)$ & $(36)$ & $(73)$ & $(27)$ & $(64)$ & $(36)$ & $(36)$ & $(64)$ \\
\hline Subtypes & & & & & & & & & \\
\hline DLBCL non & $\mathbf{2 9}$ & 17 & 12 & 16 & 13 & 13 & 16 & 16 & 13 \\
GC & & $(59)$ & $(41)$ & $(55)$ & $(45)$ & $(45)$ & $(55)$ & $(55)$ & $(45)$ \\
BCLU & $\mathbf{2}$ & 2 & 0 & 2 & 0 & 0 & 2 & 0 & 2 \\
Burkitt & $\mathbf{1}$ & 1 & 0 & 1 & 0 & 0 & 1 & 0 & 1 \\
lymphoma & & $(100)$ & & $(100)$ & & & $(100)$ & & $(100)$ \\
\hline
\end{tabular}

Table 2. There was no correlation of expression of NFATc1 with CD163 or MYC or Ki67

\begin{tabular}{|c|c|c|c|c|c|c|}
\hline \multirow{2}{*}{ NFATc1 } & \multicolumn{2}{|c|}{ CD163 } & \multicolumn{2}{c|}{ CMYC } & \multicolumn{2}{c|}{ Ki67 } \\
\cline { 2 - 7 } & Positive & Negative & Positive & Negative & Very high & High \\
\hline Positive & 8 & 5 & 9 & 8 & 6 & 8 \\
\hline p value & \multicolumn{2}{|c|}{0.800} & \multicolumn{2}{|c|}{0.505} & \multicolumn{2}{|c|}{1.000} \\
\hline
\end{tabular}

Chi square test, $p$ values $<0.05$ were considered to be statistically significant.

\section{Discussion}

Our samples(table 1) showed that $65.5 \%$ DLBCL patients were under 60 years (19 out 29 patients). In addition to this, males were slightly more than females (17 out 29; 58.6\%). Based on location, 18 out 29 (62.1\%) DLBCL cases were nodal. This findings may imply that tumor microenvironment of DLBCL nonGCB subtype was influenced by ages, gender and location (nodal versus extra nodal).

Interestingly, different from western studies, where non-GCB subtype was mostly found in older patients with the medianpatient age is in the sixth - seventh decade of life.11,12 Other study showed older ages with cut-off point 60 years correlated to higher incidence of DLBCL non-GCB subtype. 13 All DLBCL cases in our study were non-GCB subtype and among them, more patients were younger than 60 years (19 out of 29; $66 \%$ ). These findings were similar to other Asian studies.14,15 Most likely patients of our cases had unhealthy life styles since their early life and continue until theyhave been ill. It may also relate to unhealthy food consumed by patients. Their environment was may have severe contamination by pollutant. However, the information related to patient habit and jobs was 
not available.

Our cases demonstrated that males were more likely suffer from DLBCL (M:F ratio was 1.6:1). Data from WHO showed similar trend.16 The possible reason is that males are prone to pollutant exposure i.e. related to their occupation where they may use inappropriate protection. It most likely cigarette add risk of this type of lymphoma, while in Indonesia smoking habit is much higher among males than female also among younger people.17,18 Although other data showed smoking habit was correlated to risk of follicular lymphomadevelopment, 19 it might be possible DLBCL transformed from follicular lymphoma as well as de novo DLBCL pathogenesis could be related to smoking habit, further experiment should be conducted to verify this hypothesis.

Similar finding by Gascoyne et al. (2017) and Martelli et al. (2013) who found 60\% DLBCL in nodal as our data $(54.17 \%$ cases).1,11 These may because majority of DLBCL cell of origin is from germinal center B cells of lymph nodes (nodal) which expose much molecular events during their development more than other B cell types. These lead B cells are more fragile to molecular alterations i.e. translocation or gene mutation.20,21 To confirm this speculation further experiment should be performed.

$\mathrm{Ki}-67$ is an immunohistochemical marker indirectly used to evaluate the degree of lymphoma progressivity. In this study we propose that beside Ki-67, MYC and CD163 protein expression can be predictive or prognostic markers of DLBCL. While NFATc1 protein expression can be included in a diagnostic panel of a DLBCL among other markers or variables. Although our finding shows that among $56.25 \%$ subjects with $\mathrm{Ki}$ 67 expression more than $70 \%$ showed aggressive diseases, only few of them showed high expression of NFATc1 or $\mathrm{CD} 163^{+}$or MYC (table 1). However, Ki67 alone cannot be a risk factor in DLBCL patients and other factors should be considered i.e. age, sex, MYC rearrangement, EBV, HIV or other viral infection status.

Unfortunately, we do not have data of overall survival (OS) and disease-free survival (DFS) of our samples, hence, it is difficult to ensure whether protein expression of NFATc1, MYC, and CD163 have roles in prognosis. Hence, we indirectly try to connect prognosis data of protein expression of NFATc1, MYC, CD163 with proliferation index Ki-67, or DLBCL subtypes, because DLBCL non-GC type has worse prognosis. ${ }^{2}$

NFATc 1 protein is an important transcription factor in lymphomagenesis.22,23 Our finding showed the expression of NFATc1 in nuclei of lymphoma cells in a certain subset of patients (figure 1A) in line with the findings of Pham et al. (2010). ${ }^{22}$ It implies the important roles of NFATc1 in DBLCL pathogenesis specifically in non-GCB subtypes. However, we did not observe the nuclear nor cytosolic expression of NFATc1 among $\mathrm{CD} 63^{+}$macrophages. These finding may indicate that NFATc1 was functionally suppressed in TAM or may transiently activate macrophages and then NFATc1 rapidly deactivated. Other possibility is that NFATc1 might support polarization of M1 macrophages to M2like macrophages (CD163+), once $\mathrm{CD} 163^{+}$macrophages polarized, NFATc1 is deactivated. Since we did not use double positive techniques to see these possibilities and other markers for M1 macrophages (CD68), the exact possible mechanisms are still unclear i.e. cytokines/chemokine/other transcription factors or other mechanisms may contribute macrophages polarization. In line with previous data that NFATc1 has important role in macrophage activation of bone, 24 hence, it is possible that NFATc1 may activate CD163+ macrophages or polarize macrophages from M1 to CD163+TAM in lymphomas including DLBCL. These promises need further clarification. In addition, NFATc1 expression was also observed in cytoplasm of infiltrating small lymphocytes among cell tumors (figure 1A), suggesting suppression effect of NFATc1 or other NFAT family members i.e. NFATc2,25 thus, infiltrating cells (lymphocytes) at this state were most likely anergic induced by tumor cells.

Through inhibition of tumor microenvironment (TME), DLBCL patients may have alternative therapy by disrupting the activation of TME components which have important roles in lymphomagenesis or progressivity. We speculate that certain DLBCL variant 
with high density of TAM together with high expression of Ki-67 and high density of tumor cells with high expression of MYC have to be separated to be distinct sub-variant. In this study some variables i.e. OS and DFS were unknown, hence, further study should be conducted to ensure the roles of NFATc1 in DLBCL. Calcineurin/NFAT pathway most likely has an important role in lymphomagenesis of those regulation through activation of MYC and TAM-CD163+, therefore, NFATc1 can be a candidate biomarker in diagnosis or prognosis of this type of lymphoma and it could become an alternative novel immunotherapy of non-Hodgkin B cell high grade.

The expression of PD-L1 in DLBCL is low. 26 In the literature the expression of this molecule was only found in $20-27 \%$ of cases, which was mainly observed in non-GCB cases and in EBV positive DLBCL patients. 27 We tried to check this possibility by employing anti-PD-L1 antibody but PD-L1 was not expressed in all our cases, although all our cases are non-GCB subtype, but, EBV status was not confirmed. We repeated the procedure and our control was positive, but the result was still negative. It possible that this clone was not work well in lymphoid cells. Hence, for the future experiment we will employ other clones of PD-L1 and check for EBV status of patients.

Taken together, all these findings indicate that ages of patients were the most influenced factor for development and or progression of DLBCL non-GCB type. Suppression of NFATc1 expression in tumor microenvironment of DLBCL could facilitate progressivity of DLBCL. Moreover, tumor microenvironment through activation of $\mathrm{CD} 163^{+\mathrm{M} 2}$ macrophages may leads to progressivity of this type of lymphoma. NFATc1 might facilitate development and progressivity of certain subset of DLBCL non-GCB type through modulation of M2 macrophages particularly among younger male patients. In addition to these, NFATc1 may be used as a predictive or prognostic marker for certain subsets of DLBCL non-GCB type. Further study is necessary to clarify the exact roles of NFATc1 in tumor microenvironment of DLBCL.

\section{Conclusion}

NFATc1 may affect development and or progression of certain subsets of DLBCL non-GCB subtype.

\section{Acknowledgements}

We would like to thank Sri and Eka (Department of Anatomic Pathology, Faculty of Medicine Universitas Sriwijaya) for providing technical support of immunohistochemistry.

\section{Funding Statement}

This work has been supported by grants from the Hibah Penelitian Dosen Fakultas Kedokteran Universitas Sriwijaya, 2017 (99/UN9.1.4/UPPM/PL/VII/2017).

\section{References}

1. Gascoyne RD, Campo E, Jaffe ES, Chan WC, Chan JKC, Rosenwald A et al. Diffuse large Bcell lymphoma, NOS. In: Swerdlow SH, Campo E, Harris NL, Jaffe ES, PileriSA, Stein H, Thiele J., editors. WHO classification of tumours of haematopoietic and lymphoid tissues. Xxth Edition. Lyon: IARC; 2017.p. 291-97.

2. Hans CP, Weisenburger DD, Greiner TC, Gascoyne RD, Delabie J, Ott G et al. Confirmation of the molecular classification of diffuse large B-cell lymphoma by immunohistochemistry using a tissue microarray. Blood. 2004; 103: 275-82.

3. Topalian SL, Hodi FS, Brahmer JR, et al. Safety, activity, and immune correlates of antiPD-1 antibody in cancer. N Engl J Med. 2012; 366: 2443-54.

4. Woo SR, Corrales L, Gajewski TF. Innate immune recognition of cancer. Annu Rev mmunol. 2015; 33: 445-74.

5. Chanmee $\mathrm{T}$, Ontong $\mathrm{P}$, Konno $\mathrm{K}$, Itano $\mathrm{N}$. Tumor-associated macrophages as major players in the tumor microenvironment. Cancers. 2014; 6: 1670-90. 
6. Macian F, Lopez-Rodrõguez C, and Rao A. Partners in transcription: NFAT and AP-1. Oncogene. 2001; 20: 2476-89.

7. Oestreich KJ, Yoon H, Ahmed R, Boss JM. NFATc1 regulates programmed death-1 expression upon $\mathrm{T}$ cell activation. J Immunol. 2008; 181: 4832-39.

8. Vaidya R, Witzig TE. Prognostic factors for diffuse large B-cell lymphoma in the R(X)CHOP era. Ann Oncol. 2014 ;25: 2124-33.

9. Babu G, Lakshmaiah K C, Dasappa L, Babu S, Abraham LJ, Premalatha C S, Rao CR, Rajeev L K, Rudresha A H, Lokesh K N, Garg S, Agarwal A. Ki-67 and subtype as prognostic and predictive markers of diffuse large B-Cell lymphoma. Clin Cancer Investig J. 2017; 6: 97102.

10. Broyde A, Boycov O, Strenov Y, Okon E, Shpilberg O, Bairey O. Role and prognostic significance of the $\mathrm{Ki}-67$ index in nonHodgkin's lymphoma. Am J Hematol. 2009; 84: 338-43.

11. Martelli M, Ferreri AJM, Agostinelli C, Rocco AD, Pfreundschuh M, Piler SA. Diffuse large Bcell lymphoma. Crit Rev Oncol Hematol. 2013; 87: 146-71.

12. Anderson JR, Armitage JO, Weisenburger DD Epidemiology of the non-Hodgkin's lymphomas: distributions of the major subtypes differ by geographic locations. NonHodgkin's Lymphoma Classification Project. Ann Oncol. 1998; 9: 717-20.

13. Ozbalak M, CemAr M, Tuzuner N, Salihoglu A, Eskazan AE, Aydin SO, et al. Detailed analysis of diffuse large B cell lymphoma patients: A single center, retrospective study. ISRN Hematology. 2013; 2013: 275-82.

14. Shia AK, Gan GG, Jairaman S, et al. High frequency of germinal centre derivation in diffuse large B cell lymphoma from Asian patients. J Clin Pathol. 2005; 58: 962-7.

15. Shiozawa E, Yamochi-Onizuka T, Takimoto M, et al. The GCB subtype of diffuse large B-cell lymphoma is less frequent in Asian countries. Leuk Res. 2007; 31: 1579-83.

16. Ferlay J, Soerjomataram I, Ervik M, Dikshit R, Eser S, Mathers C et al. GLOBOCAN 2012 v1.0, Cancer Incidence and Mortality Worldwide: IARC Cancer Base No.11[Internet]. Lyon, France: International Agency for Research on Cancer; 2016. (Accessed April 24, 2018, at http:/ /globocan.iarc.fr.).

17. WHO Regional Office for South-East Asia. (2015). Country Profiles WHO Framework Convention on Tobacco Control in WHO SouthEast Asia Region. http://www.searo.who.int/tobacco/document s/tfi_country_profiles_2015.pdf?ua $=1$

18. Nurwidya F, Takahashi F, Baskoro H, Hidayat M, Yunus F, Takahashi K. Strategies for an effective tobacco harm reduction policy in Indonesia. Epidemiology and Health. 2014; 36:e2014035.

19. Morton LM, Hartge P, Holford TR, Holly EA, Chiu $\mathrm{BCH}$, Vineis $\mathrm{P}$ et al. Cigarette smoking and risk of non-Hodgkin lymphoma: A pooled analysis from the International Lymphoma Epidemiology Consortium (InterLymph). Cancer Epidemiol Biomarkers Prev. 2005; 14: 925-33.

20. Calado DP, Sasaki Y, Godinho SA, Pellerin A, Köchert K, Sleckman BP et al. The cell-cycle regulator $\mathrm{c}-\mathrm{Myc}$ is essential for the formation and maintenance of germinal centers. Nat. Immunol. 2012; 13: 1092-100.

21. Dominguez-Sola D, Victora GD, Ying CY, Phan RT, Saito M, Nussenzweig MC et al. The protooncogene MYC is required for selection in the germinal center and cyclic reentry. Nat. Immunol. 2012; 13:1083-91.

22. Pham LV, Tamayo AT, Li C, Bueso-Ramos C, and Ford RJ. An epigenetic chromatin remodeling role for NFATc1 in transcriptional regulation of growth and survival genes in diffuse large B-cell lymphomas. Blood. 2010; 116: 3899-906. 
23. Marafioti T, Pozzobon M, Hansmann M-L, Ventura R, Pileri SA, Roberton $\mathrm{H}$ et al. The NFATc1 transcription factor is widely expressed in white cells and translocates from the cytoplasm to the nucleus in a subset of human lymphomas. Br. J. Haematol. 2004; 128: 333-42.

24. Yasui T, Hirose J, Tsutsumi S, Nakamura K, Aburatani H, Tanaka S. Epigenetic regulation of osteoclast differentiation: possible involvement of Jmjd3 in the histone demethylation of Nfatc1. J Bone Miner Res. 2011; 26: 2665-71.

25. Shou J, Jing J, Xie J, You L, Jing Z, Han W, Pan H. Nuclear factor of activated $T$ cells in cancer development and treatment. Cancer Letters. 2015; 361: 174-84.

26. Andorsky DJ, Yamada RE, Said J, Pinkus GS, Betting DJ, and Timmerman JM. Programmed death ligand 1 is expressed by non-Hodgkin lymphomas and inhibits the activity of tumorassociated T cells. Clin Cancer Res. 2011; 17: 4232-44.

27. Quan L, Chen X, Liu A, Zhang Y, Guo X, Yan $\mathrm{S}$, et al. PD-1 Blockade Can Restore Functions of T-Cells in Epstein-Barr Virus-Positive Diffuse Large B-Cell Lymphoma In Vitro. PLoS ONE. 2015; 10 (9): e0136476.

28. Kleffel S., Posch C., Barthel SR., Mueller H., Schlapbach C., Guenova E., et al. Melanoma cell-intrinsic $\mathrm{PD}-1$ receptor functions promote tumor growth. Cell. 2015; 162: 1242-56. 